Nonlinear Processes in Geophysics, 12, 481-490, 2005

SRef-ID: $1607-7946 / \mathrm{npg} / 2005-12-481$

European Geosciences Union

(c) 2005 Author(s). This work is licensed

under a Creative Commons License.

\title{
On deterministic error analysis in variational data assimilation
}

\author{
F.-X. Le Dimet ${ }^{1}$ and V. Shutyaev ${ }^{2}$ \\ ${ }^{1}$ LMC-IMAG, Université Joseph Fourier, Grenoble, France \\ ${ }^{2}$ Institute of Numerical Mathematics, Russian Academy of Sciences, Moscow, Russia
}

Received: 21 July 2004 - Revised: 31 January 2005 - Accepted: 11 April 2005 - Published: 13 May 2005

Part of Special Issue "Quantifying predictability"

\begin{abstract}
The problem of variational data assimilation for a nonlinear evolution model is considered to identify the initial condition. The equation for the error of the optimal initialvalue function through the errors of the input data is derived, based on the Hessian of the misfit functional and the second order adjoint techniques. The fundamental control functions are introduced to be used for error analysis. The sensitivity of the optimal solution to the input data (observation and model errors, background errors) is studied using the singular vectors of the specific response operators in the error equation. The relation between "quality of the model" and "quality of the prediction" via data assimilation is discussed.
\end{abstract}

\section{Introduction}

Numerical weather prediction has been operational for several decades, numerical oceanic forecast is becoming operational, and climate prediction is a challenge for the scientific community.

The following ingredients are necessary to carry out a forecast:

- models: the geophysical fluids are governed by the laws of conservation of mass, momentum, and energy. From the mathematical point of view we get a set of nonlinear partial differential equations. These equations are of the first order with respect to time.

- observations: they are provided by in-situ measurements (e.g. the international observation system) or by remote sensing (e.g. observations from satellites). $\mathrm{Ob}-$ servations are heterogeneous both in quality and density.

Prediction is obtained by an integration of the model from an initial state at a given time. A major difficulty is to re-

Correspondence to: V. Shutyaev

(shutyaev@inm.ras.ru) trieve the initial condition from observations. A constraint is that the initial condition should verify the general properties of the atmosphere or of the ocean and therefore we cannot expect that simple interpolation will fulfil this requirement.

We can define "data assimilation" as the process mixing models and data as sources of information. Optimal control methods (e.g. Le Dimet and Talagrand, 1986) are now used in several operational centers. The basic principle is to consider the initial condition as a control variable and optimize the initial condition in order to minimize the discrepancy between the observations and the solution of the model. A major advantage of this technique is the definition of an optimality system (OS) which contains all the available information.

In practice the system includes errors of different nature:

- physical errors in the model due to approximations to be used;

- numerical errors due to the discretization of the equations and to numerical algorithms for solving the problem;

- observation errors.

Prediction should take into account all these sources of errors and it is clear that the "best" model will not lead necessarily to the "best" prediction with the same set of observations.

The purpose of this paper is to analyze the impact of errors on the prediction in order to provide some information on the improvement of prediction. This is a new statement in comparison with the former paper by Le Dimet et al. (2002b).

The paper presents the developments of the ideas of Le Dimet et al. (2002b) for the case when the original model is approximated by a difference analogue. We study the effect of the errors of the input data to the error of the optimal solution via variational data assimilation. We also study the impact of errors on the prediction, involving the case of small mesh sizes.

The paper is organized as follows. In Sect. 2, we give the statement of the variational data assimilation problem for a 
nonlinear evolution model to identify the initial condition. In Sect. 3, the equation of the error of the optimal solution is derived through the errors of the input data using the Hessian of the misfit functional. The fundamental control functions are introduced in Sect. 4 to be used for error analysis. The sensitivity of the optimal solution to the input data is studied in Sect. 5 using the singular vectors of the specific response operators in the error equation. The relation between quality of the model and quality of the prediction via data assimilation is discussed in Sect. 6. The Appendix presents the list of main notations that identifies the meanings of the variables used.

\section{Statement of the problem}

Consider the mathematical model of a physical process that is described by the evolution problem

$$
\left\{\begin{aligned}
\frac{\partial \bar{\varphi}}{\partial t} & =F(\bar{\varphi}), \quad t \in(0, T) \\
\left.\bar{\varphi}\right|_{t=0} & =\bar{u}
\end{aligned}\right.
$$

where $\bar{\varphi}=\bar{\varphi}(t)$ is the unknown function belonging for any $t$ to the Hilbert space $X, \bar{u} 1 n X, F$ is a nonlinear operator mapping $X$ into $X$. Let $Y=L_{2}(0, T ; X),\|\cdot\|_{Y}=(\cdot, \cdot)_{Y}^{1 / 2}$. Suppose that for a given $\bar{u} \in X$ there exists a unique solution $\bar{\varphi} \in Y$ to the problem (Eq. 2.1).

We will consider also the problem approximating (in some sense) Eq. (2.1) in the form:

$$
\left\{\begin{aligned}
\frac{\partial \varphi^{h}}{\partial t} & =F^{h}\left(\varphi^{h}\right), \quad t \in(0, T) \\
\left.\varphi^{h}\right|_{t=0} & =u^{h}
\end{aligned}\right.
$$

where $\quad \varphi^{h}=\varphi^{h}(t) \in X^{h}, \quad u^{h} \in X^{h}, \quad F^{h}: X^{h} \rightarrow X^{h}$, $Y^{h}=L_{2}\left(0, T ; X^{h}\right)$, and $X^{h}$ is some finite-dimensional Hilbert space (for example, a difference analogue of $X$ ). The parameter $h$ defines a set of models approximating Eq. (2.1). This parameter may be related to the mesh size of a finite-difference (or finite-element) approximation of the original problem.

Let us introduce the functional

$S\left(u^{h}\right)=\frac{\alpha}{2}\left\|u^{h}-u_{0}^{h}\right\|_{X^{h}}^{2}+\frac{1}{2}\left\|C^{h} \varphi^{h}-\varphi_{o b s}\right\|_{Y_{o b s}}^{2}$,

where $\alpha=$ const $\geq 0, u_{0}^{h} \in X^{h}$ is a prior initial-value function (background state), $\varphi_{o b s} \in Y_{o b s}$ is a prescribed function (observational data), $Y_{o b s}$ is a Hilbert space (observation space), $C^{h}: Y^{h} \rightarrow Y_{o b s}$ a linear bounded operator.

We will assume the initial-condition function $u^{h}$ to be unknown. To find it we consider the following data assimilation problem: find $u^{h}$ and $\varphi^{h}$ such that they satisfy Eq. (2.2), and on the set of solutions to Eq. (2.2), the functional $S\left(u^{h}\right)$ takes the minimum value, i.e.

$$
\left\{\begin{aligned}
\frac{\partial \varphi^{h}}{\partial t} & =F^{h}\left(\varphi^{h}\right), \quad t \in(0, T) \\
\left.\varphi^{h}\right|_{t=0} ^{0} & =u^{h} \\
S\left(u^{h}\right) & =\inf _{v \in X^{h}} S(v) .
\end{aligned}\right.
$$

Problems in the form (Eq. 2.3) were studied by Pontryagin et al. (1962); Lions (1968, 1988) (see also Agoshkov and Marchuk, 1993; Blayo et al., 1998; Glowinski and Lions, 1994; Kurzhanskii and Khapalov, 1991; Le Dimet and Talagrand, 1986; Marchuk and Penenko, 1978; Marchuk et al., 1996; Navon, 1995; Sasaki, 1970, and others).

The necessary optimality condition reduces the problem (Eq. 2.3) to the following system (Lions, 1968):

$\left\{\begin{aligned} \frac{\partial \varphi^{h}}{\partial t} & =F^{h}\left(\varphi^{h}\right), \quad t \in(0, T) \\ \left.\varphi^{h}\right|_{t=0} & =u^{h},\end{aligned}\right.$

$\left\{\begin{aligned}-\frac{\partial \varphi^{* h}}{\partial t}-\left(F^{h^{\prime}}\left(\varphi^{h}\right)\right)^{*} \varphi^{* h} & =-C^{h^{*}}\left(C^{h} \varphi^{h}-\varphi_{o b s}\right), \\ \left.\varphi^{* h}\right|_{t=T} & =0,\end{aligned}\right.$

$\alpha\left(u^{h}-u_{0}^{h}\right)-\left.\varphi^{* h}\right|_{t=0}=0$

with the unknowns $\varphi^{h}, \varphi^{* h}$, and $u^{h}$, where $\left(F^{h^{\prime}}\left(\varphi^{h}\right)\right)^{*}$ is the adjoint to the Frechet derivative of $F^{h}$, and $C^{h^{*}}$ is the adjoint to $C^{h}$ defined by $\left(C^{h} \varphi^{h}, \psi\right)_{Y_{o b s}}=\left(\varphi^{h}, C^{h^{*}} \psi\right)_{Y h}, \varphi^{h} \in$ $Y^{h}, \psi \in Y_{\text {obs }}$. We assume that the system (Eqs. 2.4-2.6) has a unique solution.

Having solved the system (Eqs. 2.4-2.6) for $t \in(0, T)$, with the initial-condition function $u^{h}$ we can make a prediction, for example, on the interval $(0,2 T)$, i.e. solve the problem (Eq. 2.2) for $t \in(0,2 T)$ and find $\left.\varphi^{h}\right|_{t=2 T}$. The question arises: how to estimate the difference between the prognostic value $\left.\varphi^{h}\right|_{t=2 T}$ and the exact (true) value $\left.\bar{\varphi}\right|_{t=2 T}$ ? Very often, the value of this difference may show a quality of the prediction: the less is the norm of the difference the better is the quality of the prediction. Another important question is what is the behaviour of the prediction error if we change one model of the form Eq. (2.2) to another (that is, with respect to $h$ ). By changing $h$, we could change a "quality of the model" ( for example, in finite-dimensional approximations, by deminishing $h$ we have usually a model of a better accuracy, i.e. of a better "quality").

Here, assuming that the solution of the problem (Eqs. 2.42.6) exists, we study the mentioned questions using the properties of the Hessian of the misfit functional and singular vectors of the specific response operators.

\section{Error equation and Hessian}

The system (Eqs. 2.4-2.6) with the three unknowns $\varphi^{h}, \varphi^{* h}, u^{h}$ may be treated as an operator equation of the form

$\mathcal{F}\left(U^{h}, U_{d}\right)=0$

where $U^{h}=\left(\varphi^{h}, \varphi^{* h}, u^{h}\right), U_{d}=\left(u_{0}^{h}, \varphi_{o b s}\right)$.

Let us project the problem Eq. (2.1) onto the difference space $X^{h}$ :

$\left\{\begin{aligned} \frac{\partial \bar{\varphi}_{h}}{\partial t} & =(F(\bar{\varphi}))_{h}, \quad t \in(0, T) \\ \left.\bar{\varphi}_{h}\right|_{t=0} & =\bar{u}_{h},\end{aligned}\right.$ 
where the lower subscript $h$ means the projection, for example, $\bar{\varphi}_{h}=(\bar{\varphi})_{h}$ is the projection of the exact solution $\bar{\varphi}$ onto $X^{h}$. This means that we consider the problem (Eq. 2.1) at mesh points.

Let us introduce the functions

$\xi_{1}^{h}=u_{0}^{h}-\bar{u}_{h}, \quad \xi_{2}^{h}=\varphi_{o b s}-C^{h} \bar{\varphi}_{h}$.

The functions $\xi_{1}^{h}$ and $\xi_{2}^{h}$ play the roles of a background error and an observation error, respectively. The problem (Eq. 3.2) may be written in the form:

$$
\left\{\begin{aligned}
\frac{\partial \bar{\varphi}_{h}}{\partial t} & =F^{h}\left(\bar{\varphi}_{h}\right)-\xi_{3}^{h}, \quad t \in(0, T) \\
\left.\bar{\varphi}_{h}\right|_{t=0} & =\bar{u}_{h},
\end{aligned}\right.
$$

where $\xi_{3}^{h}=F^{h}\left(\bar{\varphi}_{h}\right)-(F(\bar{\varphi}))_{h}$ is the approximation error of the operator $F$ on the exact solution $\bar{\varphi}$. The function $\xi_{3}^{h}$ plays the role of a model error. Together with Eq. (3.3), the following system is satisfied:

$$
\begin{gathered}
\left\{\begin{aligned}
-\frac{\partial \varphi^{*}}{\partial t}-\left(F^{h^{\prime}}\left(\bar{\varphi}_{h}\right)\right)^{*} \varphi^{*} & =-C^{h^{*}}\left(C^{h} \bar{\varphi}_{h}-C^{h} \bar{\varphi}_{h}\right), \\
\left.\varphi^{*}\right|_{t=T} & =0,
\end{aligned}\right. \\
\alpha\left(\bar{u}_{h}-\bar{u}_{h}\right)-\left.\varphi^{*}\right|_{t=0}=0
\end{gathered}
$$

with $\varphi^{*}=0$. Note that Eq. (3.4) is just like Eq. (2.5) except that $\varphi_{o b s}=C^{h} \bar{\varphi}_{h}$, which implies that that the solution in finite dimensional Hilbert space is exactly mapped to the observations. The same is true concerning Eqs. (3.5) and (2.6): Eq. (3.5) is just like Eq. (2.6) except that $u_{0}^{h}=\bar{u}_{h}$, which implies that the background state coincides with the exact solution in finite dimensional Hilbert space. We introduce these "trivial" Eqs. (3.4) and (3.5) artificially, in order to subtract then Eqs. (3.3)-(3.5) from Eqs. (2.4)-(2.6) and to obtain the system for the errors.

We have $u_{0}^{h}=\bar{u}_{h}+\xi_{1}^{h}, \varphi_{o b s}=C^{h} \bar{\varphi}_{h}+\xi_{2}^{h}, \xi_{1}^{h} \in X^{h}, \xi_{2}^{h} \in Y_{o b s}$. Let $\delta \varphi^{h}$ be an error of the model solution defined at mesh points: $\delta \varphi^{h}=\varphi^{h}-\bar{\varphi}_{h}$, and $\delta u^{h}$ be the initial-value error: $\delta u^{h}=u^{h}-\bar{u}_{h}$. Then, subtracting Eqs. (3.3)-(3.5) from Eqs. (2.4)-(2.6) and eliminating the second-order terms, we get for $\delta \varphi^{h}, \delta u^{h}$ the system:

$$
\begin{aligned}
& \left\{\begin{array}{c}
\frac{\partial \delta \varphi^{h}}{\partial t}-F^{h^{\prime}}\left(\bar{\varphi}_{h}\right) \delta \varphi^{h}=\xi_{3}^{h}, \quad t \in(0, T), \\
\left.\delta \varphi^{h}\right|_{t=0}=\delta u^{h},
\end{array}\right. \\
& \left\{\begin{array}{c}
-\frac{\partial \varphi^{* h}}{\partial t}-\left(F^{h^{\prime}}\left(\bar{\varphi}_{h}\right)\right)^{*} \varphi^{* h}=\left(F^{h^{\prime \prime}}\left(\bar{\varphi}_{h}\right) \delta \varphi^{h}\right)^{*} \varphi^{*}- \\
-C^{h^{*}}\left(C^{h} \delta \varphi^{h}-\xi_{2}^{h}\right), \\
\left.\varphi^{* h}\right|_{t=T}=0,
\end{array}\right. \\
& \alpha\left(\delta u^{h}-\xi_{1}^{h}\right)-\left.\varphi^{* h}\right|_{t=0}=0 .
\end{aligned}
$$

The problem (Eqs. 3.6-3.8) is a linear data assimilation problem; for $\varphi^{*}=0$ it is equivalent to the following minimization problem: find $u$ and $\varphi$ such that

$$
\left\{\begin{aligned}
\frac{\partial \varphi}{\partial t}-F^{h^{\prime}}\left(\bar{\varphi}_{h}\right) \varphi & =\xi_{3}^{h}, \quad t \in(0, T) \\
\left.\varphi\right|_{t=0} & =u \\
S_{1}(u) & =\inf _{v} S_{1}(v)
\end{aligned}\right.
$$

where

$$
S_{1}(u)=\frac{\alpha}{2}\left\|u-\xi_{1}^{h}\right\|_{X^{h}}^{2}+\frac{1}{2}\left\|C^{h} \varphi-\xi_{2}^{h}\right\|_{Y_{o b s}}^{2} .
$$

Consider the Hessian $H$ of the functional (Eq. 3.10); it is defined by the successive solutions of the following problems (e.g. Le Dimet et al., 2002a):

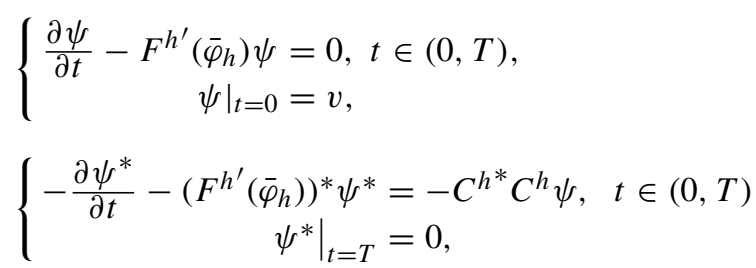

$H v=\alpha v-\left.\psi^{*}\right|_{t=0}$

Below we introduce three auxiliary operators $R_{1}, R_{2}, R_{3}$. Let $R_{1}=\alpha E$, where $E$ is the identity operator in $X^{h}$. Let us introduce the operator $R_{2}: Y_{o b s} \rightarrow X^{h}$ acting on the functions $g \in Y_{o b s}$ according to the equation

$R_{2} g=\left.\theta^{*}\right|_{t=0}$,

where $\theta^{*}$ is the solution to the adjoint problem

$\left\{\begin{aligned}-\frac{\partial \theta^{*}}{\partial t}-\left(F^{h^{\prime}}\left(\bar{\varphi}_{h}\right)\right)^{*} \theta^{*} & =C^{h^{*}} g, \quad t \in(0, T) \\ \left.\theta^{*}\right|_{t=T} & =0 .\end{aligned}\right.$

We introduce also the operator $R_{3}: Y^{h} \rightarrow X^{h}$ defined successively by the equations:

$$
\begin{aligned}
& \left\{\begin{array}{r}
\frac{\partial \theta_{1}}{\partial t}-F^{h^{\prime}}\left(\bar{\varphi}_{h}\right) \theta_{1}=q, q \in Y \\
\left.\theta_{1}\right|_{t=0}=0
\end{array}\right. \\
& \left\{\begin{array}{r}
-\frac{\partial \theta_{1}^{*}}{\partial t}-\left(F^{h^{\prime}}\left(\bar{\varphi}_{h}\right)\right)^{*} \theta_{1}^{*}=-C^{h^{*}} C^{h} \theta_{1}, \quad t \in(0, T) \\
\left.\theta_{1}^{*}\right|_{t=T}=0,
\end{array}\right. \\
& R_{3} q=\left.\theta_{1}^{*}\right|_{t=0} .
\end{aligned}
$$

From Eqs. (3.11)-(3.18) we conclude that the system (Eqs. 3.6-3.8) is equivalent to the single equation for $\delta u^{h}$ :

$H \delta u^{h}=R_{1} \xi_{1}^{h}+R_{2} \xi_{2}^{h}+R_{3} \xi_{3}^{h}$.

The Hessian $H$ acts in $X^{h}$ with domain of definition $D(H)=X^{h}$, it is self-adjoint and non-negative. If $\alpha>0$, the operator $H$ is positive definite (in the case $Y_{o b s}=Y^{h}, C^{h}=E$, the operator $H$ is positive definite even if $\alpha=0$ ). The following estimate is valid:

$(H v, v)_{X^{h}} \geq \mu_{\min }(v, v)_{X^{h}}, \quad \forall v \in X^{h}$,

where $\mu_{\min }$ is the least eigenvalue of the operator $H$, and $\mu_{\min } \geq \alpha$.

The spectrum of the operator $H$ is defined by the spectrum of the operator $C^{h^{*}} C^{h}$ through the Rayleigh relation $(H v, v) /(v, v)$ (e.g. Dunford and Schwartz, 1963). From the equality

$(H v, v)_{X^{h}}=\alpha(v, v)_{X^{h}}+\left(C^{h} \psi, C^{h} \psi\right)_{Y_{o b s}}$, 
it is easily seen that the spectrum bounds of the Hessian $H$ are defined by

$\mu_{\min }=\alpha+\inf _{v \in X^{h}} \frac{\left(C^{h^{*}} C^{h} \psi, \psi\right)_{Y_{o b s}}}{(v, v)_{X^{h}}}$,

$\mu_{\max }=\alpha+\sup _{v \in X^{h}} \frac{\left(C^{h^{*}} C^{h} \psi, \psi\right)_{Y_{o b s}}}{(v, v)_{X^{h}}}$,

where $\psi$ is the solution to Eq. (3.11).

Thus, the operator $C^{h^{*}} C^{h}$ plays an important role for the spectrum of the operator $H$. If the operator $C^{h^{*}} C^{h}$ is ill-conditioned, then the operator $H$ may also be illconditioned, and this should be taken into account when solving Eq. (3.19).

In the case when $F^{h^{\prime}}\left(\bar{\varphi}_{h}\right)=A$ is a $t$-independent operator, the Eqs. (3.11)-(3.13) imply

$\psi=e^{A t}, \quad \psi^{*}=-\int_{t}^{T} e^{-A^{*}(t-s)} C^{h^{*}} C^{h} \psi d s$,

$H v=\alpha v+\int_{0}^{T} e^{A^{*} s} C^{h^{*}} C^{h} e^{A s} v d s, v \in X^{h}$

Hence,

$H=\alpha E+\int_{0}^{T} e^{A^{*} s} C^{h^{*}} C^{h} e^{A s} d s$.

Therefore, in this case, the operator $C^{h^{*}} C^{h}$ defines the Hessian $H$ explicitly.

As follows from Eq. (3.19), the error $\delta u^{h}$ of the optimal solution depends on the errors $\xi_{1}^{h}, \xi_{2}^{h}, \xi_{3}^{h}$ linearly and continuously. The influence of the errors $\xi_{1}^{h}, \xi_{2}^{h}, \xi_{3}^{h}$ on the value of $\delta u^{h}$ is determined by the operators $H^{-1} R_{1}, H^{-1} R_{2}, H^{-1} R_{3}$, respectively. The values of the norms of these operators may be considered as an influence criteria: the less is the norm of the operator $H^{-1} R_{i}$, the less impact on $\delta u^{h}$ is given by the corresponding error $\xi_{i}^{h}$. This criteria may be used also to choose the regularization parameter $\alpha$ (Tikhonov et al., 1995; Morozov, 1987).

\section{Fundamental control functions for error analysis}

Since the model (Eq. 2.2) is finite-dimensional, the Hessian $H$ has a complete orthonormal system in $X^{h}$ of eigenfunctions $v_{k}$ corresponding to the eigenvalues $\mu_{k}$ :

$H v_{k}=\mu_{k} v_{k}$

where $\left(v_{k}, v_{l}\right)_{X}=\delta_{k l}, \quad k, l=1,2, \ldots$, and $\delta_{k l}$ is the Kronecker delta. The singular value decomposition of $H$ coincides with the eigenvalue decomposition: $H=U D U^{*}$, where $D=\operatorname{diag}\left(\mu_{1}, \mu_{2}, \ldots\right)$ is a diagonal martix, and $U$ is the matrix of eigenvectors of $H$.
It is easily seen that the eigenvalue problem (Eq. 4.1) is equivalent to the system:

$\left\{\begin{aligned} \frac{\partial \varphi_{k}}{\partial t}-F^{h^{\prime}}\left(\bar{\varphi}_{h}\right) \varphi_{k} & =0, t \in(0, T), \\ \left.\varphi_{k}\right|_{t=0} & =v_{k},\end{aligned}\right.$
$\left\{\begin{aligned}-\frac{\partial \varphi_{k}^{*}}{\partial t}-\left(F^{h^{\prime}}\left(\bar{\varphi}_{h}\right)\right)^{*} \varphi_{k}^{*} & =-C^{h^{*}} C^{h} \varphi_{k}, \quad t \in(0, T) \\ \left.\varphi_{k}^{*}\right|_{t=T} & =0,\end{aligned}\right.$

$\alpha v_{k}-\left.\varphi_{k}^{*}\right|_{t=0}=\mu_{k} v_{k}$

By the analogy with the Poincaré-Steklov operator theory (Lebedev and Agoshkov, 1983), we say that the system of functions $\left\{\varphi_{k}, \varphi_{k}^{*}, v_{k}\right\}$ satisfying (Eqs. 4.2-4.4) is the system of "fundamental control functions". For data assimilation problems, these functions were introduced by Shutyaev (1995).

Using the fundamental control functions, we can obtain the solution of the error Eq. (3.19) in the explicit form. Equation (3.19) is equivalent to the system (Eqs. 3.6-3.8) and may be written as the following system:

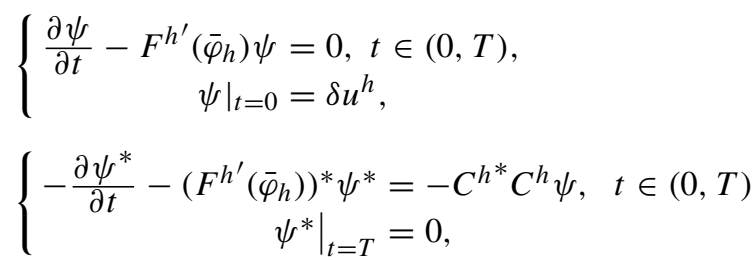

$\alpha \delta u^{h}-\left.\psi^{*}\right|_{t=0}=P$,

where $P=R_{1} \xi_{1}^{h}+R_{2} \xi_{2}^{h}+R_{3} \xi_{3}^{h}$ is the right-hand side of Eq. (3.19).

The solution $\psi, \psi^{*}, \delta u^{h}$ of the system (Eqs. 4.5-4.7) may be represented in the form:

$\psi=\sum_{k} a_{k} \varphi_{k}, \quad \psi^{*}=\sum_{k} a_{k} \varphi_{k}^{*}, \quad \delta u^{h}=\sum_{k} a_{k} v_{k}$,

where $\varphi_{k}, \varphi_{k}^{*}, v_{k}$ are the fundamental control functions defined by Eqs. (4.2)-(4.4), $a_{k}=\left(P, v_{k}\right)_{X^{h}} / \mu_{k}$.

From Eq. (4.8), we have the representation for the Fourier coefficients $\left(\delta u^{h}\right)_{k}$ of the error $\delta u^{h}$ :

$\left(\delta u^{h}\right)_{k}=a_{k}=\frac{1}{\mu_{k}}\left(R_{1} \xi_{1}^{h}+R_{2} \xi_{2}^{h}+R_{3} \xi_{3}^{h}, v_{k}\right)_{X^{h}}$.

Note that

$\left(R_{1} \xi_{1}^{h}, v_{k}\right)_{X^{h}}=\alpha\left(\xi_{1}^{h}, v_{k}\right)_{X^{h}}$.

By definition of $R_{2}, R_{3}$,

$\left(R_{2} \xi_{2}^{h}, v_{k}\right)_{X^{h}}=\left(\left.\theta^{*}\right|_{t=0}, v_{k}\right)_{X^{h}}$,

$\left(R_{3} \xi_{3}^{h}, v_{k}\right)_{X^{h}}=\left(\left.\theta_{1}^{*}\right|_{t=0}, v_{k}\right)_{X^{h}}$,

where $\theta^{*}$ is the solution of Eq. (3.15) for $g=\xi_{2}^{h}$, and $\theta_{1}, \theta_{1}^{*}$ are the solutions of Eqs. (3.16)-(3.17) for $q=\xi_{3}^{h}$. From Eqs. (3.15) and (4.2) we get

$\left(\left.\theta^{*}\right|_{t=0}, v_{k}\right)_{X^{h}}=\left(C^{h^{*}} \xi_{2}^{h}, \varphi_{k}\right)_{Y^{h}}$. 
Hence,

$\left(R_{2} \xi_{2}^{h}, v_{k}\right)_{X^{h}}=\left(\xi_{2}^{h}, C^{h} \varphi_{k}\right)_{Y_{o b s}}$.

Analogously, from Eqs. (3.17) and (4.2),

$\left(\left.\theta_{1}^{*}\right|_{t=0}, v_{k}\right)_{X^{h}}=\left(-\theta_{1}, C^{h^{*}} C^{h} \varphi_{k}\right)_{Y^{h}}$.

Further, Eqs. (3.16) and (4.3) give

$\left(\theta_{1},-C^{h^{*}} C^{h} \varphi_{k}\right)_{Y^{h}}=\left(\xi_{3}^{h}, \varphi_{k}^{*}\right)_{Y^{h}}$.

Hence,

$\left(R_{3} \xi_{3}^{h}, v_{k}\right)_{X^{h}}=\left(\xi_{3}^{h}, \varphi_{k}^{*}\right)_{Y^{h}}$.

From Eqs. (4.9)-(4.12) we obtain the expression for the Fourier coefficients $\left(\delta u^{h}\right)_{k}$ of the error $\delta u$ of the optimal solution through the errors $\xi_{1}^{h}, \xi_{2}^{h}, \xi_{3}^{h}$ :

$$
\begin{aligned}
& \left(\delta u^{h}\right)_{k}=\frac{\alpha}{\mu_{k}}\left(\xi_{1}^{h}, v_{k}\right)_{X^{h}}+\frac{1}{\mu_{k}}\left(\xi_{2}^{h}, C^{h} \varphi_{k}\right)_{Y_{o b s}}+ \\
& +\frac{1}{\mu_{k}}\left(\xi_{3}^{h}, \varphi_{k}^{*}\right)_{Y^{h}},
\end{aligned}
$$

where $\left\{\varphi_{k}, \varphi_{k}^{*}, v_{k}\right\}$ are the fundamental control functions defined by Eqs. (4.2)-(4.4).

Expression (4.13) shows that the fundamental control functions play the role of "sensitivity functions"; they are the weight-functions for the corresponding errors $\xi_{1}^{h}, \xi_{2}^{h}, \xi_{3}^{h}$ in the representation (4.13). Note that the fundamental control functions $\left\{\varphi_{k}, \varphi_{k}^{*}, v_{k}\right\}$ do not depend on the structure of the errors $\xi_{1}^{h}, \xi_{2}^{h}, \xi_{3}^{h}$ and may be calculated beforehand for each $k$ if necessary.

For self-adjoint $t$-independent operator $F^{h^{\prime}}\left(\bar{\varphi}_{h}\right)$, the fundamental control functions may be found in the explicit form (Shutyaev, 1995). The application of fundamental control functions to the data assimilation problem in hydrology, and some numerical results are given by Le Dimet et al. (2002b).

\section{Singular vectors and error analysis}

In this section, we study the sensitivity of the optimal initialvalue function to the input data using the singular vectors of the specific response operators in the error Eq. (3.19). We may rewrite Eq. (3.19) as

$\delta u^{h}=H^{-1} R_{1} \xi_{1}^{h}+H^{-1} R_{2} \xi_{2}^{h}+H^{-1} R_{3} \xi_{3}^{h}$.

Hence,

$\left\|\delta u^{h}\right\|_{X^{h}} \leq\left\|T_{1} \xi_{1}^{h}\right\|_{X^{h}}+\left\|T_{2} \xi_{2}^{h}\right\|_{X^{h}}+\left\|T_{3} \xi_{3}^{h}\right\|_{X^{h}}$,

where $T_{i}=H^{-1} R_{i}, T_{1}: X^{h} \rightarrow X^{h}, T_{2}: Y_{o b s} \rightarrow X^{h}, T_{3}:$ $Y^{h} \rightarrow X^{h}$.

Each summand in Eq. (5.2) determines the impact given by the correspoding error $\xi_{i}$. We have

$$
\left\{\begin{array}{l}
\left\|T_{1} \xi_{1}^{h}\right\|_{X^{h}} \leq \sqrt{\left\|T_{1}^{*} T_{1}\right\|}\left\|\xi_{1}^{h}\right\|_{X^{h}}, \\
\left\|T_{2} \xi_{2}^{h}\right\|_{X^{h}} \leq \sqrt{\left\|T_{2}^{*} T_{2}\right\|}\left\|\xi_{2}^{h}\right\|_{Y_{o b s}}, \\
\left\|T_{3} \xi_{3}^{h}\right\|_{X^{h}} \leq \sqrt{\left\|T_{3}^{*} T_{3}\right\|}\left\|\xi_{3}^{h}\right\|_{Y^{h}},
\end{array}\right.
$$

where $T_{1}^{*}: X^{h} \rightarrow X^{h}, T_{2}^{*}: X^{h} \rightarrow Y_{o b s}, T_{3}^{*}: X^{h} \rightarrow Y^{h}$ are the adjoints to $T_{i}, i=1,2,3$, and the $i$-th inequality becomes an equality when $\xi_{i}^{h}$ is the singular vector of $T_{i}$ corresponding to the largest singular value $\sigma_{\max }^{2}=\left\|T_{i}^{*} T_{i}\right\|$. The values $r_{i}=\sqrt{\left\|T_{i}^{*} T_{i}\right\|}$ may be considered as "sensitivity coefficients" which clearly demonstrate the measure of influence of the corresponding error upon the optimal solution. The higher the relative sensitivity coefficient, the more effectual is the error in question.

From Eqs. (5.1)-(5.3), we have

$$
\begin{aligned}
& \left\|\delta u^{h}\right\|_{X^{h}} \leq \sqrt{\left\|T_{1}^{*} T_{1}\right\|}\left\|\xi_{1}^{h}\right\|_{X^{h}}+\sqrt{\left\|T_{2}^{*} T_{2}\right\|}\left\|\xi_{2}^{h}\right\|_{Y_{o b s}}+ \\
& +\sqrt{\left\|T_{3}^{*} T_{3}\right\|}\left\|\xi_{3}^{h}\right\|_{Y^{h}}=r_{1}\left\|\xi_{1}^{h}\right\|_{X^{h}}+r_{2}\left\|\xi_{2}^{h}\right\|_{Y_{o b s}}+r_{3}\left\|\xi_{3}^{h}\right\|_{Y^{h}} .
\end{aligned}
$$

The Hessian $H$ defined by Eqs. (3.11)-(3.13) has a complete orthonormal system in $X$ of eigenfunctions $v_{k}$ corresponding to the eigenvalues $\mu_{k}: H v_{k}=\mu_{k} v_{k},\left(v_{k}, v_{l}\right)_{X}=\delta_{k l}$, $k, l=1,2, \ldots$ The least eigenvalue $\mu_{\min }$ of $H$ is positive if $\alpha>0$. We also assume that it is positive for $\alpha=0$ (it is true, for example, in the case $Y_{o b s}=Y^{h}, C^{h}=E$ ).

Consider the operator $T_{1}$. Since $T_{1}=H^{-1} R_{1}=\alpha H^{-1}=T_{1}^{*}$, the singular vectors of $T_{1}$ are the eigenvectors $v_{i}$ of the Hessian $H$, and the corresponding sensitivity coefficient is equal to

$r_{1}=\sqrt{\left\|T_{1}^{*} T_{1}\right\|}=\frac{\alpha}{\mu_{\min }}$.

Note that $r_{1} \leq 1$ (since $\mu_{\min } \geq \alpha$ ).

For the operator $T_{2}: Y_{o b s} \rightarrow X^{h}$ the following statement is valid (Le Dimet et al., 2002b). The singular values $\sigma_{k}^{2}$ and the corresponding orthonormal (right) singular vectors $w_{k} \in Y_{o b s}$ of the operator $T_{2}$ are defined by

$\sigma_{k}^{2}=\frac{\mu_{k}-\alpha}{\mu_{k}^{2}}, \quad w_{k}=\frac{1}{\sqrt{\mu_{k}-\alpha}} C^{h} \varphi_{k}$,

where $\mu_{k}$ are the eigenvalues of the Hessian $H$, and $\varphi_{k}$ are the fundamental control functions defined by Eq. (4.2). The left singular vectors of $T_{2}$ coincide with the eigenvectors $v_{k}$ of $H$ :

$T_{2} T_{2}^{*} v_{k}=\sigma_{k}^{2} v_{k}, \quad k=1,2, \ldots$

Thus, the sensitivity coefficient $r_{2}=\sqrt{\left\|T_{2}^{*} T_{2}\right\|}$ is defined by the equation:

$r_{2}=\max _{k} \frac{\sqrt{\mu_{k}-\alpha}}{\mu_{k}}$.

The equality $\left\|T_{2} \xi_{2}\right\|_{X^{h}}=r_{2}\left\|\xi_{2}^{h}\right\|_{Y_{o b s}}$ holds if $\xi_{2}=w_{k_{0}}$, where $w_{k_{0}}$ is the singular vector of $T_{2}$ corresponding to the largest singular value $\sigma_{k_{0}}^{2}=r_{2}^{2}$. If $\alpha=0$, the sensitivity coefficient $r_{2}$ is defined by the equation

$r_{2}=\frac{1}{\sqrt{\mu_{\min }}}$, 
where $\mu_{\min }$ is the least eigenvalue of the Hessian $H$. Note that $\mu_{\min }$ depends on $h$, and very often, in practice, $H$ is illconditioned such that $\mu_{\min } \rightarrow 0$ as $h \rightarrow 0$. In this case, $r_{2} \rightarrow \infty$, and the error may be very large.

Consider now the operator $T_{3}=H^{-1} R_{3}$. To determine the sensitivity coefficient $r_{3}=\sqrt{\left\|T_{3}^{*} T_{3}\right\|}$, we need to derive $R_{3}^{*}$. For $q \in Y^{h}, p \in X^{h}$, we have from Eqs. (3.16)-(3.18):

$\left(R_{3} q, p\right)_{X^{h}}=\left(\left.\theta_{1}^{*}\right|_{t=0}, p\right)_{X^{h}}=$

$=-\left(C^{h^{*}} C^{h} \theta_{1}, \phi\right)_{Y^{h}}=-\left(C^{h} \theta_{1}, C^{h} \phi\right)_{Y_{o b s}}$,

where $\theta_{1}, \theta_{1}^{*}$ are the solutions to Eqs. (3.16)-(3.17), and $\phi$ is the solution to Eq. (3.11) with $v=p$. Further,

$\left(R_{3} q, p\right)_{X^{h}}=-\left(\theta_{1}, C^{h^{*}} C^{h} \phi\right)_{Y^{h}}=\left(q, \phi^{*}\right)_{Y^{h}}$

and $R_{3}^{*} p=\phi^{*}$, where $\phi^{*}$ is the solution to the adjoint problem:

$$
\left\{\begin{aligned}
-\frac{\partial \phi^{*}}{\partial t}-\left(F^{h^{\prime}}\left(\bar{\varphi}_{h}\right)\right)^{*} \phi^{*} & =-C^{h^{*}} C^{h} \phi, \quad t \in(0, T) \\
\left.\phi^{*}\right|_{t=T} & =0 .
\end{aligned}\right.
$$

The operator $R_{3} R_{3}^{*}: X^{h} \rightarrow X^{h}$ may be defined as follows: for given $p \in X^{h}$ find $\phi$ as the solution of Eq. (3.11) with $v=p$, find $\phi^{*}$ as the solution of Eq. (5.9), and for $q=\phi^{*}$ find $\theta_{1}, \theta_{1}^{*}$ as the solutions of Eqs. (3.16)-(3.17); then put $R_{3} R_{3}^{*} p=\left.\theta_{1}^{*}\right|_{t=0}$.

Therefore, the operator $T_{3} T_{3}^{*}=H^{-1} R_{3} R_{3}^{*} H^{-1}$ is defined by the successive solutions of the following problems (for given $\left.v \in X^{h}\right)$ :

$H p=v$,

$\left\{\begin{aligned} \frac{\partial \phi}{\partial t}-F^{h^{\prime}}\left(\bar{\varphi}_{h}\right) \phi & =0, t \in(0, T), \\ \left.\phi\right|_{t=0} & =p,\end{aligned}\right.$

$\left\{\begin{aligned}-\frac{\partial \phi^{*}}{\partial t}-\left(F^{h^{\prime}}\left(\bar{\varphi}_{h}\right)\right)^{*} \phi^{*} & =-C^{h^{*}} C^{h} \phi, \quad t \in(0, T) \\ \left.\phi^{*}\right|_{t=T} & =0 .\end{aligned}\right.$

$\left\{\begin{aligned} \frac{\partial \theta_{1}}{\partial t}-F^{h^{\prime}}\left(\bar{\varphi}_{h}\right) \theta_{1} & =\phi^{*}, \quad t \in(0, T) \\ \left.\theta_{1}\right|_{t=0} & =0,\end{aligned}\right.$

$\left\{\begin{aligned}-\frac{\partial \theta_{1}^{*}}{\partial t}-\left(F^{h^{\prime}}\left(\bar{\varphi}_{h}\right)\right)^{*} \theta_{1}^{*} & =-C^{h^{*}} C^{h} \theta_{1}, \quad t \in(0, T) \\ \left.\theta_{1}^{*}\right|_{t=T} & =0,\end{aligned}\right.$

$H w=\left.\theta_{1}^{*}\right|_{t=0}$

then

$T_{3} T_{3}^{*} v=w$,

and we have for the sensitivity coefficient $r_{3}$ :

$r_{3}=\sqrt{\left\|T_{3} T_{3}^{*}\right\|}$

The algorithm (Eqs. 5.10-5.17) can be used to compute the sensitivity coefficient $r_{3}$ numerically.

\section{Specific case}

To compare the sensitivity coefficients $r_{1}, r_{2}, r_{3}$ we consider a specific case. Suppose $Y_{o b s}=Y^{h}, \quad C^{h}=E$ (the identity operator), and $F^{h^{\prime}}\left(\bar{\varphi}_{h}\right)=\left(F^{h^{\prime}}\left(\bar{\varphi}_{h}\right)\right)^{*}=-A$ is a $t$ independent self-adjoint operator such that $A$ generates an orthonormal basis in $X^{h}$ consisting of eigenfunctions $v_{k}$ : $A v_{k}=\lambda_{k} v_{k}, \quad\left(v_{k}, v_{j}\right)_{X}=\delta_{k j}$, where $\lambda_{k}$ are the corresponding eigenvalues of $A$. Then the eigenfunctions of the Hessian $H$ coincide with the eigenfunctions $v_{k}$, and the eigenvalues $\mu_{k}$ are defined by Shutyaev (1995):

$\mu_{k}=\alpha+\frac{1-e^{-2 \lambda_{k} T}}{2 \lambda_{k}}$.

(Note that if $\lambda_{k}=0$, we put $\mu_{k}=\alpha+T$, by continuity.) In this case

$\mu_{\min }=\alpha+\frac{1-e^{-2 \lambda_{\max } T}}{2 \lambda_{\max }}$,

where $\lambda_{\max }$ is the largest eigenvalue of $A$. From Eqs. (5.5) and (5.8), we get

$r_{1}=\alpha\left(\alpha+\frac{1-e^{-2 \lambda_{\max } T}}{2 \lambda_{\max }}\right)^{-1}$,

$r_{2}=\max _{k} \sqrt{\frac{1-e^{-2 \lambda_{k} T}}{2 \lambda_{k}}}\left(\alpha+\frac{1-e^{-2 \lambda_{k} T}}{2 \lambda_{k}}\right)^{-1}$.

To find $r_{3}$ consider the operator $T_{3} T_{3}^{*}$ defined by the Eqs. (5.10)-(5.17). For $v=v_{k}$ we get

$p=\frac{1}{\mu_{k}} v_{k}, \phi=\frac{1}{\mu_{k}} e^{-\lambda_{k} t} v_{k}, \phi^{*}=-\frac{1}{\mu_{k}} e^{\lambda_{k} t} \int_{t}^{T} e^{-2 \lambda_{k} s} v_{k} d s$,

$\theta_{1}=-\frac{1}{\mu_{k}} e^{-\lambda_{k} t} \int_{0}^{t} e^{2 \lambda_{k} \xi} \int_{\xi}^{T} e^{-2 \lambda_{k} s} v_{k} d s d \xi$,

$\theta_{1}^{*}=\frac{1}{\mu_{k}} \int_{t}^{T} e^{-\lambda_{k}(\theta-t)} e^{-\lambda_{k} \theta} \int_{0}^{\theta} e^{2 \lambda_{k} \xi} \int_{\xi}^{T} e^{-2 \lambda_{k} s} v_{k} d s d \xi d \theta$,

$w=\left.\frac{1}{\mu_{k}} \theta_{1}^{*}\right|_{t=0}=\frac{1}{\mu_{k}^{2}} \int_{0}^{T} e^{-2 \lambda_{k} \theta} \int_{0}^{\theta} e^{2 \lambda_{k} \xi} \int_{\xi}^{T} e^{-2 \lambda_{k} s} v_{k} d s d \xi d \theta$

Then, from Eq. (5.16),

$T_{3} T_{3}^{*} v_{k}=\sigma_{k}^{2} v_{k}, \quad k=1,2, \ldots$,

where

$\sigma_{k}^{2}=\frac{1}{\mu_{k}^{2}} \int_{0}^{T} e^{-2 \lambda_{k} \theta} \int_{0}^{\theta} e^{2 \lambda_{k} \xi} \int_{\xi}^{T} e^{-2 \lambda_{k} s} d s d \xi d \theta=$ 


$$
\begin{aligned}
& =\frac{1}{\mu_{k}^{2}} \int_{0}^{T} e^{2 \lambda_{k} \xi}\left(\int_{\xi}^{T} e^{-2 \lambda_{k} s} d s\right)^{2} d \xi= \\
& =\frac{1}{\left(2 \mu_{k} \lambda_{k}\right)^{2}}\left(\frac{1-e^{-4 \lambda_{k} T}}{2 \lambda_{k}}-2 T e^{-2 \lambda_{k} T}\right)>0 .
\end{aligned}
$$

Thus, $v_{k}$ are the left singular values of $T_{3}$ corresponding to the singular values $\sigma_{k}^{2}$. The right singular vectors of $T_{3}$ satisfy the equation

$T_{3}^{*} T_{3} w_{k}=\sigma_{k}^{2} w_{k}, \quad k=1,2, \ldots$

and are defined by

$w_{k}=\frac{1}{\sigma_{k}} T_{3}^{*} v_{k}$

Due to Eqs. (4.2)-(4.3),

$w_{k}=\frac{1}{\sigma_{k}} R_{3}^{*} H^{-1} v_{k}=\frac{1}{\sigma_{k} \mu_{k}} R_{3}^{*} v_{k}=\frac{1}{\sigma_{k} \mu_{k}} \varphi_{k}^{*}$

and

$\left(w_{k}, w_{l}\right)_{Y}=\frac{1}{\sigma_{k} \mu_{k} \sigma_{l} \mu_{l}}\left(\varphi_{k}^{*}, \varphi_{l}^{*}\right)_{Y^{h}}=\frac{1}{\sigma_{k} \mu_{k} \sigma_{l} \mu_{l}} \times$

$\times\left(R_{3}^{*} v_{k}, R_{3}^{*} v_{l}\right)_{Y^{h}}=\frac{1}{\sigma_{k} \sigma_{l}}\left(H^{-1} R_{3} R_{3}^{*} H^{-1} v_{k}, v_{l}\right)_{X^{h}}=$

$=\frac{1}{\sigma_{k} \sigma_{l}}\left(T_{3} T_{3}^{*} v_{k}, v_{l}\right)_{X^{h}}=\frac{\sigma_{k}}{\sigma_{l}}\left(v_{k}, v_{l}\right)_{X^{h}}=\delta_{k l}$

Therefore, the singular values $\sigma_{k}^{2}$ of the operator $T_{3}$ are defined by the equation:

$\sigma_{k}^{2}=\frac{1}{\left(2 \mu_{k} \lambda_{k}\right)^{2}}\left(\frac{1-e^{-4 \lambda_{k} T}}{2 \lambda_{k}}-2 T e^{-2 \lambda_{k} T}\right)$,

where $\mu_{k}=\alpha+\left(1-e^{-2 \lambda_{k} T}\right) /\left(2 \lambda_{k}\right)$. The left singular vectors of $T_{3}$ coincide with the eigenvectors $v_{k}$, and the corresponding orthonormal right singular vectors $w_{k} \in Y$ are defined as

$w_{k}=\frac{1}{\sigma_{k} \mu_{k}} \varphi_{k}^{*}, \quad \varphi_{k}^{*}(t)=\frac{1}{2 \lambda_{k}} e^{\lambda_{k} t}\left(e^{-2 \lambda_{k} T}-e^{-2 \lambda_{k} t}\right) v_{k}$,

where $\varphi_{k}^{*}$ are the fundamental control functions satisfying Eqs. (4.2)-(4.4). Thus, the sensitivity coefficient $r_{3}=\sqrt{\left\|T_{3}^{*} T_{3}\right\|}$ is defined by the equation:

$r_{3}=\max _{k} \frac{1}{2 \mu_{k}\left|\lambda_{k}\right|} \sqrt{\frac{1-e^{-4 \lambda_{k} T}}{2 \lambda_{k}}-2 T e^{-2 \lambda_{k} T}}$.

From Eqs. (6.1), (6.3), one can derive the typical behaviour of the sensitivity coefficients $r_{1}, r_{2}, r_{3}$, depending on the parameter $\alpha$ and the mesh size $h$. Let us compare the coefficients $r_{1}, r_{2}$, and $r_{3}$. It is easily seen that

$r_{1} \leq 1, \quad r_{2} \leq \frac{1}{2 \sqrt{\alpha}}$

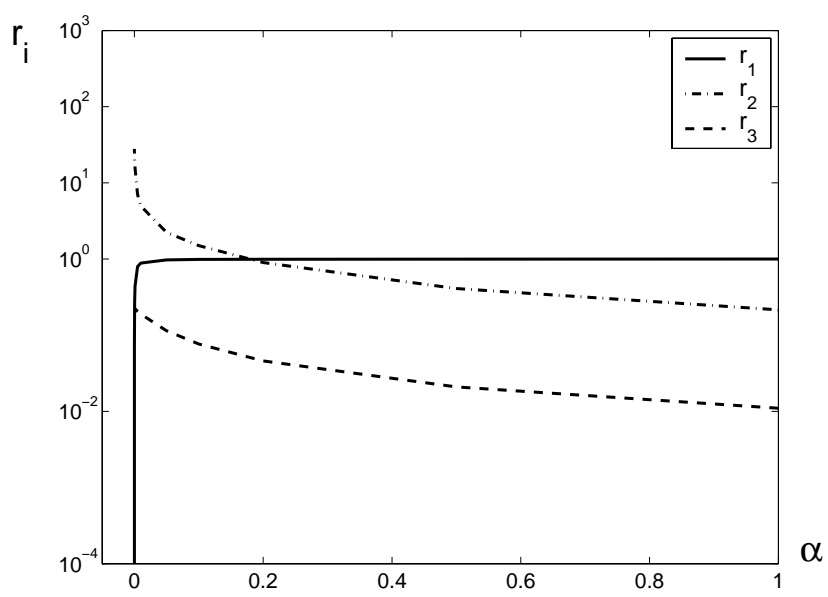

Fig. 1. Sensitivity coefficients $r_{i}, i=1,2,3$, for $h=10^{-1}$.

Moreover, $r_{1} \rightarrow 0$ as $\alpha \rightarrow 0$, whereas $r_{2}$ may be very large, because $r_{2} \leq\left(\sqrt{\mu_{\min }}\right)^{-1}$ and for $\alpha=0$ we have

$r_{2}=\frac{1}{\sqrt{\mu_{\min }}}=\sqrt{\frac{2 \lambda_{\max }}{1-e^{-2 \lambda_{\max } T}}}$,

and if $\lambda_{\max } \rightarrow \infty$ as $h \rightarrow 0$, then $r_{2} \rightarrow \infty$.

For $r_{3}$, from Eq. (6.3) we can conclude that $r_{3}$ is always bounded even if $\alpha \rightarrow 0$. For $\alpha=0$ we have

$r_{3}=\max _{k}\left|1-e^{-2 \lambda_{k} T}\right|^{-1} \sqrt{\frac{1-e^{-4 \lambda_{k} T}}{2 \lambda_{k}}-2 T e^{-2 \lambda_{k} T}}$.

Since the function

$f(\lambda)=\left(1-e^{-2 \lambda T}\right)^{-2}\left(\frac{1-e^{-4 \lambda T}}{2 \lambda}-2 T e^{-2 \lambda T}\right)$

is bounded for $\lambda \in(-\infty,+\infty)$, the sensitivity coefficient $r_{3}$ will be bounded when $h \rightarrow 0$.

So, with the regularization parameter $\alpha>0$, all the coefficients $r_{1}, r_{2}, r_{3}$ are bounded even if $h \rightarrow 0$. The situation differs when $\alpha \rightarrow 0$. If $\alpha=0$, then $r_{1}=0, r_{3}$ is bounded, but $r_{2}$ may be very large (as $h \rightarrow 0$ ). This is demonstrated by Figs. 1-2 which present the sensitivity coefficients versus the parameter $\alpha$ with $h=10^{-1}$ and $h=10^{-2}$ for the case when $T=1$ and $\lambda_{k}$ are an approximation of the eigenvalues of the 1D Laplace operator given by the equation (Lebedev and Agoshkov, 1983): $\lambda_{k}=\frac{4}{h^{2}} \sin ^{2} \frac{\pi k h}{2}, \quad k=1,2, \ldots, n-1, \quad h=1 / n$. The figures show that for small $\alpha$ the optimal solution is most sensitive to the observation error.

Such a typical behaviour of the sensitivity coefficients $r_{1}, r_{2}, r_{3}$ was also noticed in more general cases. Some numerical computations of sensitivity coefficients for the data assimilation problem in hydrology are given by Le Dimet et al. (2002b).

Thus, the sensitivity of the optimal solution to the input errors is determined by the value of the sensitivity coefficients 


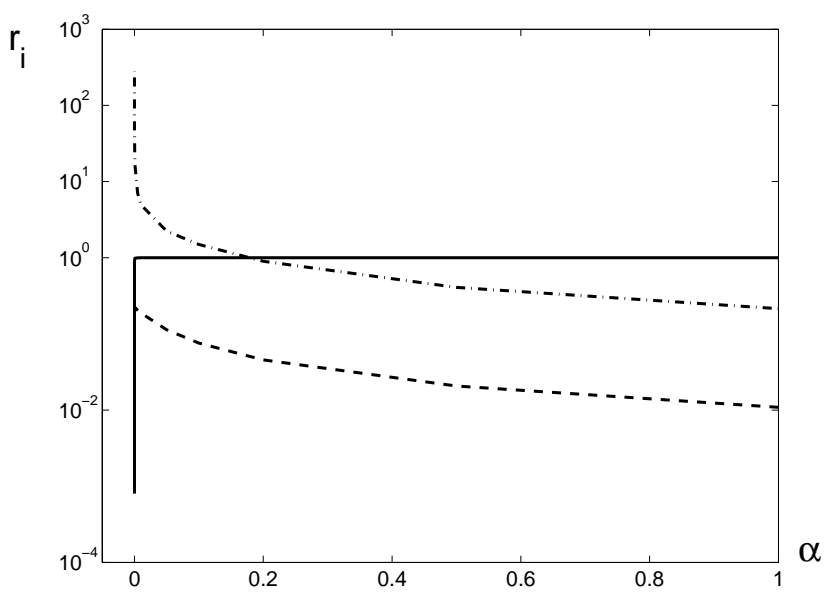

Fig. 2. Sensitivity coefficients $r_{i}, i=1,2,3$, for $h=10^{-2}$.

which are the norms of the specific response operators relating the error of the input to the error of the optimal initialvalue function. The maximum error growth for the output is given by the singular vectors of the corresponding response operator. The singular vectors are the fundamental control functions; they form complete orthonormal systems in specific functional spaces and may be used for error analysis.

Remark. We have considered a constant weight on the initial condition penalty in the objective function $S$. This implies white noise. It is not a limitation of the method, it is a simplification for ease of presentation. In practice, in variational assimilation, "regularization" is achieved through a whole covariance matrix, not only a numerical coefficient $\alpha$. In this case, the Hessian will involve the covariance matrix instead of $\alpha$, and equations for the sensitivity coefficients $r_{1}, r_{2}, r_{3}$ will be more complicated, defined through the Hessian and this covariance matrix.

\section{Prediction error}

Having solved the system (Eqs. 2.4-2.6) for $t \in(0, T)$, with the initial-condition function $u^{h}$ we can make a prediction on the interval $(0,2 T)$, i.e. solve the problem (Eq. 2.2) for $t \in(0,2 T)$ and find $\left.\varphi^{h}\right|_{t=2 T}$. To estimate the difference between the prognostic value $\left.\varphi^{h}\right|_{t=2 T}$ and the exact (true) value $\left.\bar{\varphi}\right|_{t=2 T}$ we can use the results of Sect. 5 concerning the error $\delta u^{h}=u^{h}-\bar{u}_{h}$.

Having solved Eq. (3.19), we find $\delta u^{h}=u^{h}-\bar{u}_{h}$. To predict the error $\delta \varphi^{h}=\varphi^{h}-\bar{\varphi}_{h}$ at $t=2 T$, we should solve the problem (Eq. 3.6) for $t \in(0,2 T)$. Since the model (Eq. 2.3) is finitedimensional, the following apriori estimate is valid:

$$
\left\|\delta \varphi^{h}\right\|_{\tilde{Y}^{h}}+\left\|\left.\delta \varphi^{h}\right|_{t=2 T}\right\|_{X^{h}} \leq \tilde{c}_{1}\left(\left\|\delta u^{h}\right\|_{X^{h}}+\left\|\xi_{3}^{h}\right\|_{\tilde{Y}^{h}}\right),
$$

where $\tilde{c}_{1}=$ const $>0, \quad \tilde{Y}^{h}=L_{2}\left(0,2 T ; X^{h}\right)$. Taking into account Eq. (5.4), we get

$$
\left\|\left.\varphi^{h}\right|_{t=2 T}-\left.\bar{\varphi}_{h}\right|_{t=2 T}\right\|_{X^{h}} \leq
$$

$$
\begin{aligned}
& \leq \tilde{c}_{1}\left(r_{1}\left\|\xi_{1}^{h}\right\|_{X^{h}}+r_{2}\left\|\xi_{2}^{h}\right\|_{Y_{o b s}}+r_{3}\left\|\xi_{3}^{h}\right\|_{Y^{h}}+\left\|\xi_{3}^{h}\right\|_{\tilde{Y}^{h}}\right) \leq \\
& \leq \tilde{c}_{1}\left(r_{1}\left\|\xi_{1}^{h}\right\|_{X^{h}}+r_{2}\left\|\xi_{2}^{h}\right\|_{Y_{o b s}}+\left(r_{3}+1\right)\left\|\xi_{3}^{h}\right\|_{\tilde{Y}^{h}}\right),
\end{aligned}
$$

where $r_{1}, r_{2}, r_{3}$ are the sensitivity coefficients defined by Eqs. (5.5), (5.8), (5.17), respectively.

Due to Eq. (7.2), the sensitivity coefficients $r_{1}, r_{2}, r_{3}$ show the effect of the errors $\xi_{1}, \xi_{2}, \xi_{3}$ on the prediction error $\delta \varphi^{h}$. One can use Eq. (7.2) to study the behaviour of the error $\left.\varphi^{h}\right|_{t=2 T}-\left.\bar{\varphi}_{h}\right|_{t=2 T}$ with respect to the quality of the model (i.e. with respect to $h$ ).

Since $\xi_{3}^{h}=F^{h}\left(\bar{\varphi}_{h}\right)-(F(\bar{\varphi}))_{h}$ is the approximation error of the operator $F$ on the exact solution $\bar{\varphi}$, the following estimate may be supposed to be valid:

$\left\|\xi_{3}^{h}\right\|_{\tilde{Y}^{h}} \leq c_{2} h^{k}, \quad k>0, \quad c_{2}=$ const $>0$.

To illustrate the estimate (Eq. 7.2), consider the specific cases from Sect. 6. From the results of Sect. 6, we can conclude that with the regularization parameter $\alpha>0$, all the coefficients $r_{1}, r_{2}, r_{3}$ are bounded (even if $h \rightarrow 0$ ). Since $\left(r_{3}+1\right)\left\|\xi_{3}^{h}\right\|_{\tilde{Y} h} \rightarrow 0$ as $h \rightarrow 0$, the prediction error $\left.\varphi^{h}\right|_{t=2 T}-\left.\bar{\varphi}_{h}\right|_{t=2 T}$ will be determined by $\xi_{1}^{h}$ and $\xi_{2}^{h}$. If $\xi_{1}^{h}, \xi_{2}^{h} \rightarrow 0$ as $h \rightarrow 0$, then $\left.\varphi^{h}\right|_{t=2 T}-\left.\bar{\varphi}_{h}\right|_{t=2 T}$ will also go to zero with $h \rightarrow 0$. So, the prediction error is stable with respect to the errors $\xi_{1}, \xi_{2}, \xi_{3}$ if $\alpha>0$.

The situation changes when $\alpha \rightarrow 0$. Without regularization, when $\alpha=0$, we have $r_{1}=0, r_{3}$ is bounded, but $r_{2}$ may be very large (as $h \rightarrow 0$ ). For $h \rightarrow 0$, we have again $\left(r_{3}+1\right)\left\|\xi_{3}^{h}\right\|_{\tilde{Y}^{h} \rightarrow 0 \text {, }}$ so the prediction error is stable with respect to $\xi_{3}^{h}$. However, this error may be unstable with respect $\xi_{2}^{h}:$ if $\left\|\xi_{2}^{h}\right\|_{Y_{o b s}} \rightarrow 0$ as $h \rightarrow 0$, it does not mean that $r_{2}\left\|\xi_{2}^{h}\right\|_{Y_{o b s}} \rightarrow 0$, because $r_{2}$ may go to $\infty$. This is one more reason to introduce a regularization when solving the original data assimilation problem.

The above analysis shows that the prediction error is most sensitive to the observation errors. Therefore, the effect of the observation errors in data assimilation is of great importance and should be taken into account when solving the problem.

\section{Conclusions}

The impact of the input errors on the prediction via data assimilation is due to the error of the optimal initial-value function. The sensitivity of the optimal solution to the input errors may be determined by the value of the sensitivity coefficients which are the norms of the specific response operators relating the error of the input to the error of the optimal initialvalue function. The maximum error growth for the output is given by the singular vectors of the corresponding response operator. The singular vectors are the fundamental control functions which form complete orthonormal systems in specific functional spaces and may be used for error analysis. The prediction error is most sensitive to the observation errors, which effect is of great importance and should be taken into account when solving the problem. 


\section{Appendix: List of main notations}

$\bar{\varphi}$ is the exact solution of the original model

$\bar{u}$ is the exact initial-value function

$F$ is a nonlinear operator of the model

$t$ is time variable

$T$ is the length of the time interval

$\varphi^{h}$ is an approxomation of the model solution

$u^{h}$ is an approximation of the initial-value function

$F^{h}$ is an approximation of the nonlinear operator $F$

$X$ is a Hilbert space with the scalar product $(\cdot, \cdot)_{X}$

$X^{h}$ is a difference analogue of $X$

$Y=L_{2}(0, T ; X)$ is the space of abstract functions $f(t)$ with values in $X$; the scalar product and the norm are given by

$(f, g)_{Y}=\int_{0}^{T}(f(t), g(t))_{X} d t, \quad\|f\|_{Y}=(f, f)_{Y}^{1 / 2}$

$\varphi_{o b s}$ is the observational-data function (observation vector)

$u_{0}^{h}$ is the background state

$Y_{\text {obs }}$ is an observation space

$C^{h}$ is an observation operator

$S$ is the objective (cost) function

$C^{h^{*}}$ is the operator adjoint to $C^{h}$

$\alpha$ is a regularization parameter

$\bar{\varphi}_{h}$ is the projection of the exact solution onto $X^{h}$

$\bar{u}_{h}$ is the projection of the exact initial function onto $X^{h}$

$\xi_{1}^{h}$ is a background error

$\xi_{2}^{h}$ is an observation error

$\xi_{3}^{h}$ is a model error

$\delta \varphi^{h}$ is the error of the model solution: $\delta \varphi^{h}=\varphi^{h}-\bar{\varphi}_{h}$

$\delta u^{h}$ is the initial-value error: $\delta u^{h}=u^{h}-\bar{u}_{h}$

$H$ is the Hessian of the linear data assimilation problem

$\mu_{k}$ are the eigenvalues of the Hessian $H$

$v_{k}$ are the eigenfunctions of the Hessian $H$

$\varphi_{k}, \varphi_{k}^{*}, v_{k}$ are the system of fundamental control functions

$r_{i}$ are sensitivity coefficients defined by $r_{i}=\sqrt{\left\|T_{i}^{*} T_{i}\right\|}$

$T_{i}$ are response operators, $i=1,2,3$.

Acknowledgements. This work was carried out within the IDOPT project (CNRS, INRIA, UJF, INPG) and the project 04-05-64481 of the Russian Foundation for the Basic Research and within the programme ECO-NET (EGIDE).

We would like to express our gratitude to O. Talagrand and to the referees for their valuable suggestions and helpful comments which led to the improvement of the paper.

Edited by: O. Talagrand

Reviewed by: two referees

\section{References}

Agoshkov, V. I. and Marchuk, G. I.: On solvability and numerical solution of data assimilation problems, Russ. J. Numer. Analys. Math. Modelling, 8, 1-16, 1993.

Blayo, E., Blum, J., and Verron, J.: Assimilation variationnelle de données en océanographie et réduction de la dimension de l'espace de contrôle, Équations aux Dérivées Partielles et Applications, Elsevier, Paris, 205-219, 1998.

Cacuci, D. G.: Sensitivity theory for nonlinear systems: II. Extensions to additional classes of responses, J. Math. Phys., 22, 2803-2812, 1981.

Chavent, G.: Local stability of the output least square parameter estimation technique, Math. Appl. Comp., 2, 3-22, 1983.

Dontchev, A. L.: Perturbations, Approximations and Sensitivity Analysis of Optimal Control Systems, Lecture Notes in Control and Information Sciences, 52, Springer, Berlin, 1983.

Dunford, N. and Schwartz, J.: Linear Operators, Part II. Spectral Theory. Self Adjoint Operators in Hilbert Space, Interscience Publishers, New York, 1963.

Glowinski, R. and Lions, J. L.: Exact and approximate controllability for distributed parameter systems, Acta Numerica, 1, 269298, 1994.

Kravaris, C. and Seinfeld, J. H.: Identification of parameters in distributed parameter systems by regularization, SIAM J. Control and Optimization, 23, 217-241, 1985.

Kurzhanskii, A. B. and Khapalov, A. Yu.: An observation theory for distributed-parameter systems, Math. Syst. Estimat. Control, 1, 389-440, 1991.

Lebedev, V. I. and Agoshkov, V. I.: Poincaré-Steklov Operators and their Applications to Analysis, Depart. Comp. Math. USSR Acad. Sci., Moscow, 1983.

Le Dimet, F. X. and Charpentier, I.: Méthodes du second ordre en assimilation de données, Équations aux Dérivées Partielles et Applications, Elsevier, Paris, 623-639, 1998.

Le Dimet, F. X., Navon, I. M., and Daescu, D. N.: Second-order information in data assimilation, Month. Wea. Rev., 130, 629648, 2002a.

Le Dimet, F. X., Ngnepieba, P., and Shutyaev, V.: On error analysis in data assimilation problems, Russ. J. Numer. Anal. Math. Modelling, 17, 71-97, $2002 \mathrm{~b}$.

Le Dimet, F. X. and Talagrand, O.: Variational algorithms for analysis and assimilation of meteorological observations: theoretical aspects, Tellus, 38A, 97-110, 1986.

Lions, J. L.: Contrôle Optimal des Systèmes Gouvernés par des Équations aux Dérivées Partielles, Dunod, Paris, 1968.

Lions, J. L.: Contrôlabilité Exacte Perturbations et Stabilisation de Systèmes Distribués, Masson, Paris, 1988.

Marchuk, G. I. and Penenko, V. V.: Application of optimization methods to the problem of mathematical simulation of atmospheric processes and environment, Modelling and Optimization of Complex Systems, Proc. of the IFIP-TC7 Working conf., Springer, New York, 240-252, 1978.

Marchuk, G. I., Agoshkov, V. I., and Shutyaev, V. P.: Adjoint Equations and Perturbation Algorithms in Nonlinear Problems, CRC Press Inc., New York, 1996.

Morozov, V. A.: Regular Methods for Solving the Ill-Posed Problems, Nauka, Moscow, 1987.

Navon, I. M.: Variational data assimilation, optimal parameter estimation and sensitivity analysis for environmental problems Computational Mechanics'95, Springer, New York, 1, 740-746, 1995. 
Ngodock, H. E.: Assimilation de données et analyse de sensibilité: une application à la circulation océanique, Thèse de l'Université Joseph Fourier, UJF, Grenoble, 1995.

Pontryagin, L. S., Boltyanskii, V. G., Gamkrelidze, R. V., and Mischenko, E. F.: The Mathematical Theory of Optimal Processes, John Wiley, New York, 1962.

Sasaki, Y. K.: Some basic formalisms in numerical variational analysis, Month. Wea. Rev., 98, 857-883, 1970.
Shutyaev, V. P.: Some properties of the control operator in the problem of data assimilation and iterative algorithms, Russ. J. Numer. Analys. Math. Modelling, 10, 357-371, 1995.

Tikhonov, A. N., Leonov, A. S., and Yagola, A. G.: Nonlinear Inverse Problems, Nauka, Moscow, 1995. 